\title{
Effet du mode d'introduction aux limites des ondes de marée sur un modèle local haute définition
}

\author{
Christelle Herry, Pascal Lazure, Franck Dumas \\ IFREMER/DYNECO/PHYSED, BP 70, 29280 Plouzané
}

\begin{abstract}
$\underline{\text { Résumé }}$
Le travail suivant vise à développer une méthode permettant de reproduire précisément la marée dans les modèles hydrodynamiques locaux haute résolution. Habituellement, les conditions aux limites des modèles locaux sont générées par un modèle de plus grande emprise. Mais la précision des calculs à la côte se dégrade dans certains secteurs. Aussi, on envisage ici de corriger les conditions aux limites ouvertes des modèles locaux en utilisant les résultats plus précis issus d'une base de données (CST France ${ }^{3}$ ). Cette modification permet d'obtenir une hauteur de marée plus juste dans l'ensemble modèle de détail. De plus, elle ne perturbe pas de manière trop importante d'autres phénomènes qu'on souhaite aussi reproduire: phénomènes météorologiques et circulation résiduelle. L'utilisation de conditions aux limites modifiées semble donc tout à fait adaptée à la reproduction fidèle de la marée à la côte.
\end{abstract}

\section{$\underline{\text { Abstract }}$}

This survey focuses on the boundary conditions used to force high-resolution local hydrodynamic models. Usually, they are generated by a larger model. However results are degraded in some coastal zones. That is why we have tried to modify open boundary conditions thanks to more precise data from CST France ${ }^{3}$. This modification results in an improvement of the predicted tide in the entire local model. Moreover, it does not affect other phenomenon: weather effects or residual currents.

\section{Mots-clés}

Modèle hydrodynamique, conditions aux limites, marée, surcotes

\section{Introduction}

Le laboratoire de Physique Hydrodynamique et SEDimentaire de l'Institut Français de Recherche pour l'Exploitation de la Mer a développé un modèle hydrodynamique de l'océan côtier appelé MARS. Ce modèle, implanté dans différentes stations côtières de l'institut, est utilisé depuis trois ans comme outil de connaissance (amélioration de la perception de l'environnement littoral) et comme outil d'ingénierie pour les études d'environnement (étude d'impact et de suivi des rejets polluants, choix d'implantation d'ouvrages...). Dans le but d'améliorer les 
résultats de ce modèle, on se propose ici de s'intéresser au calcul des conditions aux limites utilisées par les modèles locaux haute résolution.

\section{Le modèle et les conditions aux limites utilisées}

Le travail réalisé porte sur la version à deux dimensions horizontales du modèle MARS. Ce modèle permet notamment de calculer, à chaque instant, les hauteurs d'eau et les courants moyens (intégrés sur la verticale). Pour cela, il s'appuie sur le système de Saint-Venant (équations primitives intégrées selon la verticale) qu'il résout sur une grille $\mathrm{C}$ (grille de discrétisation dans laquelle les vitesses sont calculées sur les bords des mailles et les autres grandeurs au centre) à pas constant en utilisant un schéma numérique de type «Implicite en Directions Alternées (A.D.I) ». Les valeurs de la pression et du vent de surface (vent à $10 \mathrm{~m}$ au-dessus de la mer) nécessaires au calcul des hauteurs d'eau et du courant sont fournies par un modèle météorologique indépendant. Lors de cette étude, on a utilisé la base de données ERA40 ${ }^{4}$ (sorties du modèle européen ECMWF validées par les conditions météorologiques observées). Enfin, le forçage aux limites du modèle se fait uniquement sur le niveau $\zeta$ de la surface libre et les valeurs des vitesses aux frontières sont déterminées dans les conditions de gradient nul.

Comme l'illustre la figure 1, les conditions aux limites sont obtenues grâce à un emboîtement de modèles. Le modèle de plus grande emprise (dit modèle de rang 0 ) a une résolution d'environ $5 \mathrm{~km}$ $\left(\mathrm{d} \varphi=0.05^{\circ} ; \mathrm{dg}=0.0833^{\circ}\right)$ et il recouvre l'ensemble du plateau européen du Portugal à l'Islande soit une zone s'étendant de $40^{\circ} \mathrm{N}$ à $65^{\circ} \mathrm{N}$ et de $20^{\circ} \mathrm{W}$ à $15^{\circ} \mathrm{E}$. Une composition harmonique de la marée ( 8 ondes) utilisant les constantes données par un modèle de marée mondial $\left(\mathrm{FES}^{2}{ }^{2}\right.$ ) permet de calculer l'élévation de la surface libre aux limites de ce modèle qui calcule les conditions aux limites des modèles de détail.

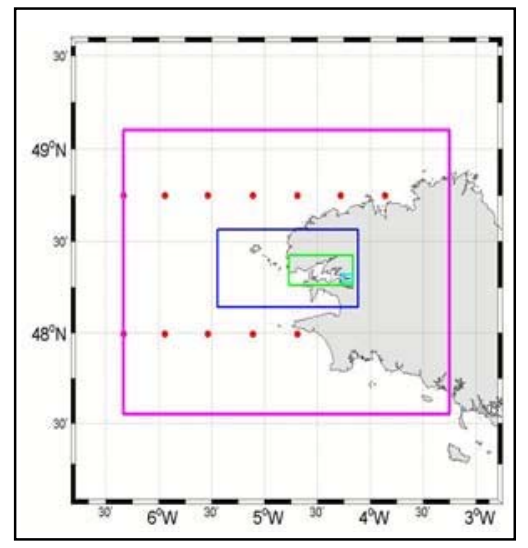

Figure 1 : Emboîtement des modèles

\section{Présentation d'une méthode corrective}

Les hauteurs de marée calculées par le modèle montrent des biais plus ou moins importants selon les régions. Ainsi, dans la baie du Mont Saint Michel où les résultats sont peu satisfaisants on calcule une erreur de marnage (moyenne sur un mois de simulation) de $72 \mathrm{~cm}$ à Cancale. Plusieurs causes peuvent expliquer ces résultats: une mauvaise représentation de la bathymétrie, un coefficient de frottement mal adapté, des approximations numériques... On a cherché à les corriger à l'aide de constantes harmoniques calculées pour 115 ondes de marée (du niveau moyen jusqu'aux ondes $6^{\text {ème }}$ diurne) par le Modèle CST France ${ }^{3}$ 


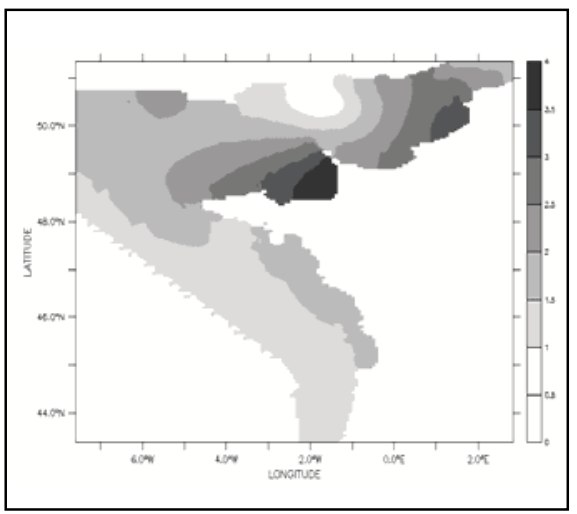

Figure 2 : Exemple de données CST France : amplitude l'onde M2 (m) (source: Service Hydrographique et Océanographique de la Marine). Il s'agit d'un modèle à deux dimensions qui intègre des hauteurs d'eau mesurées (marégraphes) en Manche et sur le plateau continental du Golfe de Gascogne. Les constantes qu'il fournit sur cette zone permettent de reconstituer des hauteurs de marée sur le plateau continental français avec une précision inégalée. (cf. figure 2)

Il ne s'agit pas ici de forcer directement le modèle de détail avec les hauteurs d'eau calculées à partir des constantes harmoniques de CST France mais de modifier uniquement la composante d'origine astronomique, $\chi_{m}$ de la hauteur d'eau calculée par le rang 0 tout en conservant le niveau moyen du modèle $\left(\mathrm{h}_{0}=0\right)$ et la surcote éventuelle, S. Pour cela, on modifie la hauteur d'eau initialement calculée par le rang 0 à la limite du modèle régional :

$$
\chi=h_{0}+\chi_{m}+S
$$

suivant la relation $(2)$ :

$$
\begin{aligned}
& \chi^{\prime}=\chi_{m}+\underbrace{\sum_{k=8}^{115} A_{c s t}^{k} \cos \left(\omega_{k}+\varphi_{c s t}^{k}\right)}-\underbrace{\sum_{k=8}^{115} A_{\text {rang } 0}^{k} \cos \left(\omega_{k}+\varphi_{\text {rang } 0}^{k}\right)}+S
\end{aligned}
$$

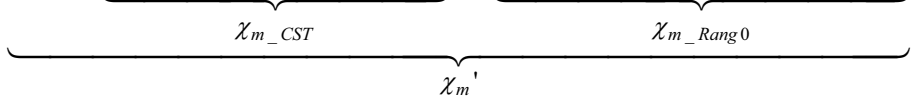

où :

- $A_{c s t}^{k}$ et $\varphi_{c s t}^{k}$ sont les constantes harmoniques (amplitude et phase) fournies par

CST France qui permettent de reconstituer la hauteur de marée $\chi_{m_{-}} C S T$.

- $A_{\text {rang } 0}^{k}$ et $\varphi_{\text {rang0 }}^{k}$ sont des constantes harmoniques de la hauteur de marée $\chi_{m_{-} \text {Rang } 0} \approx \chi_{m}$ calculée par le rang 0 . Ces constantes sont obtenues par analyse harmonique d'une simulation d'un an réalisée en marée réelle et sans forçage météorologique avec le rang 0 du modèle.

- $\chi_{m}{ }^{\prime}$ est la nouvelle hauteur de marée utilisée à la limite du rang de détail.

La composition décrite par la relation (2) ne fait pas intervenir les 7 premières ondes de marée qui sont pourtant disponibles. En effet, la première onde correspond au niveau moyen que l'on souhaite conserver et les 6 suivantes aux ondes basses fréquences (annuelles, mensuelles...). On ne souhaite pas introduire celles-ci dans le modèle car elles risquent, en perturbant le niveau moyen journalier, de perturber la circulation résiduelle dans le modèle régional (rang 1). En effet, ces ondes sont en grande partie d'origine météorologique et non purement astronomique. 


\section{Résultats : validation de la marée}

On a d'abord cherché à vérifier l'intérêt d'utiliser des conditions aux limites modifiées pour améliorer la représentation de la marée. Pour cela, on s'est intéressé aux résultats obtenus, pour une simulation d'un mois en condition de marée réelle mais sans forçage météorologique, avec les deux types de conditions aux limites envisagés (hauteurs modifiées et non modifiées), dans différents modèles régionaux. Les résultats obtenus dans les deux cas sont comparés aux hauteurs calculées à partir des données CST France. Pour cela, on a calculé la différence de marnage (cf. figure 3 ) et le déphasage moyens (sur la durée totale de la simulation) entre les deux signaux de marée comparés.

On a notamment enregistré les hauteurs calculées par le modèle en différents points situés le long de radiales reliant la limite ouest du modèle de rang 1 à la côte. Dans le cas du modèle de la rade de Brest, dont on présente les résultats ici (cf. figure 3) on a choisi un première radiale à la latitude $48^{\circ} \mathrm{N}$ et une seconde à $48,75^{\circ} \mathrm{N}$ (cf. figure 1 ).

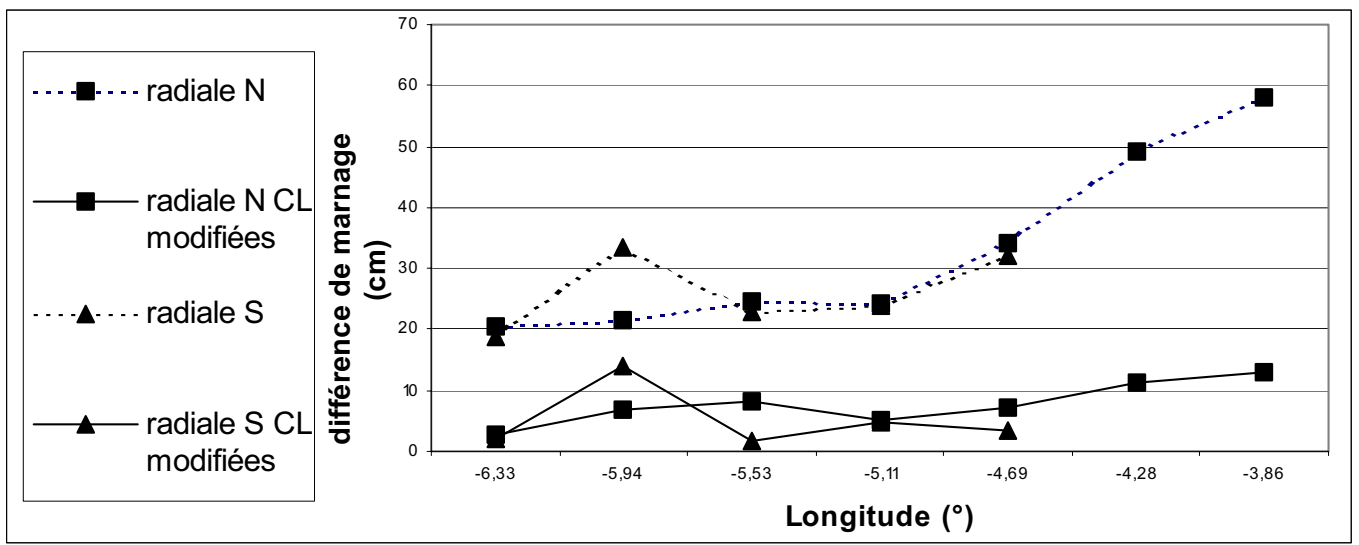

Figure 3 : Ecart de marnage dans le long des deux radiales du rang 1 du modèle « rade »

Le premier point $\left(6,33^{\circ} \mathrm{W}\right)$ des courbes de la figure 3 est situé sur la limite du rang 1 de « rade ». On peut a priori s'étonner du fait que l'erreur obtenue en ce point ne soit pas nulle lorsqu'on substitue les hauteurs de marée calculées par le rang 0 par les hauteurs issues de CST France, puisqu'en théorie ici $\chi^{\prime}=\chi_{m_{-} C S T}$. En réalité,

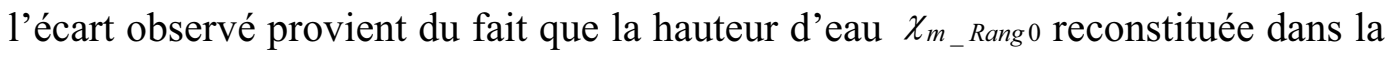
relation (2) n'est pas tout à fait identique à la hauteur $\chi_{m}$ calculée instantanément par le rang 0 . Cette différence provient certainement de phénomènes locaux tels que des oscillations naturelles (seiches) qui ne sont pas pris en compte dans l'analyse harmonique réalisée. Malgré cela, l'erreur baisse ici de $20 \mathrm{~cm}$ à $3 \mathrm{~cm}$, l'amélioration obtenue à la limite est donc importante (elle peut l'être encore d'avantage dans d'autres domaines). On constate également que, si l'écart entre les résultats du modèle et les données du SHOM augmente parfois lorsqu'on s'éloigne de la limite, l'amélioration obtenue à la limite (une réduction d'environ 
$17 \mathrm{~cm}$ sur l'écart de marnage) est conservée à l'intérieur du modèle de rang 1. Elle semble même avoir tendance à augmenter dans le cas de ce modèle particulier puisque (pour la radiale située à $48.75^{\circ} \mathrm{N}$ ) 1'erreur qui était à l'origine de presque $60 \mathrm{~cm}$ (au plus près de la cote : $3.86^{\circ} \mathrm{W}$ ) avant modification est inférieure à $15 \mathrm{~cm}$ après : ce qui fait une amélioration de $45 \mathrm{~cm}$ à la côte contre $17 \mathrm{~cm}$ sur la limite.

Enfin, on s'est également intéressé aux résultats obtenus à la côte (dans quelques ports des différents modèles testés). Pour reprendre l'exemple de Cancale cité précédemment, la différence de marnage est maintenant de $13 \mathrm{~cm}$ seulement, ce qui fait une amélioration des résultats supérieure à $80 \%$. Plus généralement, l'amélioration obtenue par la nouvelle méthode de calcul des conditions aux limites proposée est comprise, selon les ports, entre 45 et $90 \%$ à la fois en terme de différence de marnage et de déphasage moyen.

\section{Calcul des surcotes}

On appelle surcote, ou décote s'il est négatif, l'écart entre la hauteur d'eau réelle et la marée astronomique prédite. Cet écart est généré par les conditions météorologiques: pression atmosphérique et tension du vent. Les surcotes s'établissent sur des échelles spatiales de plusieurs centaines de kilomètres. Aussi, elles ne peuvent être correctement représentées que par des modèles de grande emprise ou par des modèles régionaux forcés par des conditions aux limites qui intègrent la surcote. C'est pourquoi, on a voulu vérifier les capacités des modèles de détail à reproduire ces surcotes notamment lorsqu'ils sont forcés par les conditions aux limites modifiées.

On dispose de relevés de hauteurs d'eau effectués par le Service Hydrographique et Océanographique de la Marine dans différents ports français de la Manche et de l'Atlantique entre le $1^{\mathrm{er}}$ janvier 1991 et le 31 décembre 2000. Pour la validation des résultats du modèle, on a retenu une période de six mois du $1^{\mathrm{er}}$ décembre 1999 au 31 mai 2000. Une telle période permet de tester le modèle dans différentes conditions météorologiques. D'autre part celle-ci est particulièrement intéressante car elle englobe les tempêtes de la fin décembre 1999 qui ont généré des surcotes importantes.

Les surcotes sont obtenues en filtrant les mesures de hauteurs d'eau comme les résultats du modèle (obtenus pour une simulation avec forçage météorologique) à l'aide d'un filtre Démerliac ${ }^{1}$. Ce filtre linéaire passe bas est un moyen simple et efficace pour supprimer la majorité des ondes de marée. En enlevant ensuite le niveau moyen on obtient une bonne estimation de la surcote. La figure 4 donne un exemple de résultats obtenus dans le port de Saint Gildas avec le modèle de Penbé (dont le rang 1 recouvre en partie le sud de la Bretagne et la Loire Atlantique). 


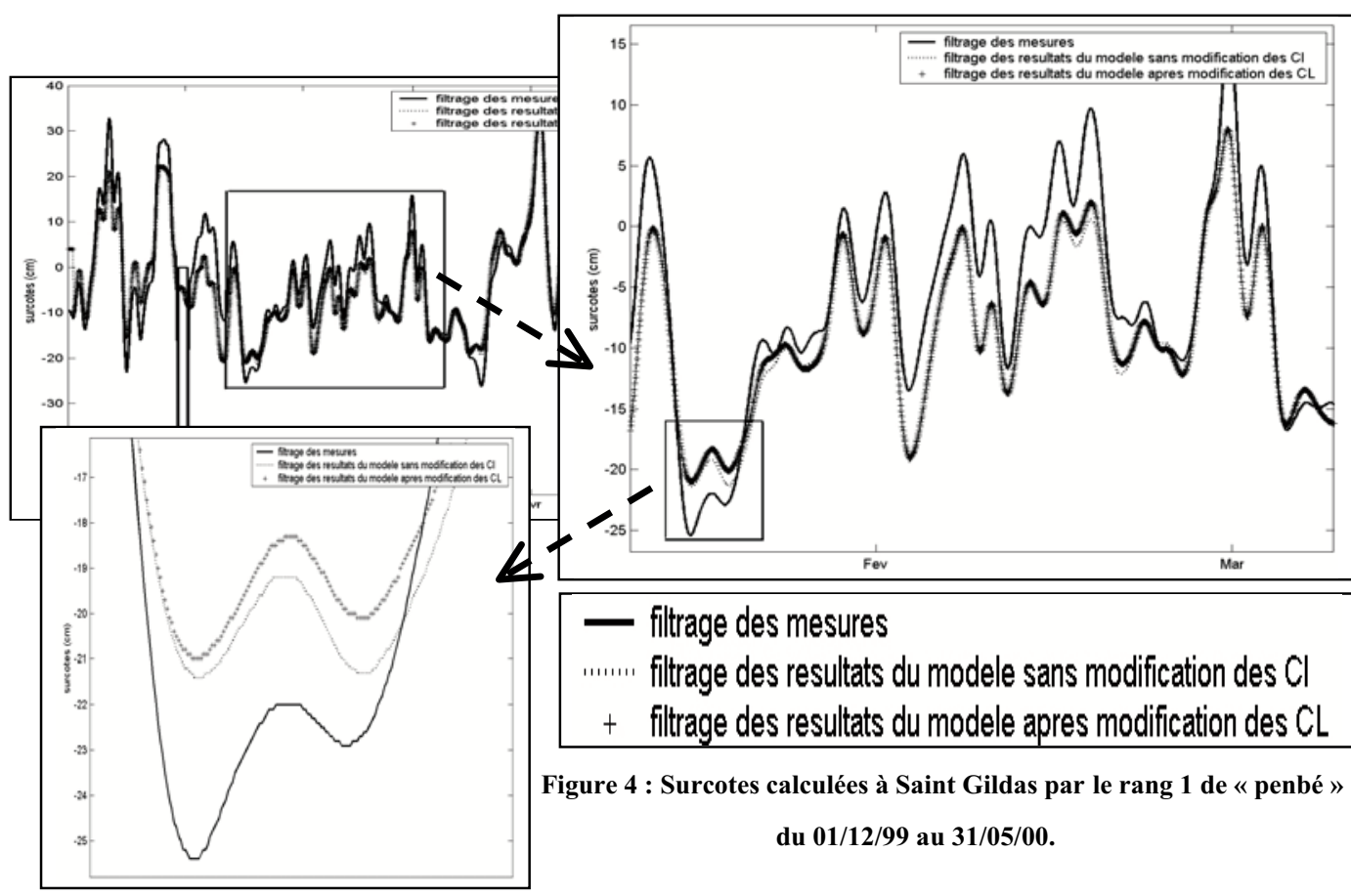

On constate que, quelles que soient les conditions utilisées aux limites du rang 1 , le modèle permet d'obtenir une bonne représentation de la surcote réelle même s'il a parfois tendance à la sous-estimer. La différence entre les résultats obtenus avec les conditions initiales et modifiées est très faible : 2 à 3 centimètres tout au plus. Il semble donc que, comme on l'espérait, la méthode utilisée pour remplacer les composantes de marée n'ait pas affecté la modélisation des phénomènes météorologiques.

\section{Calcul des courants résiduels}

Enfin, on s'est intéressé à la circulation résiduelle dans les modèles de détail. Pour cela, on trace les courants résiduels obtenus en appliquant un filtre Démerliac ${ }^{1}$ aux vitesses instantanées calculées lors d'une simulation en marée réelle seule.

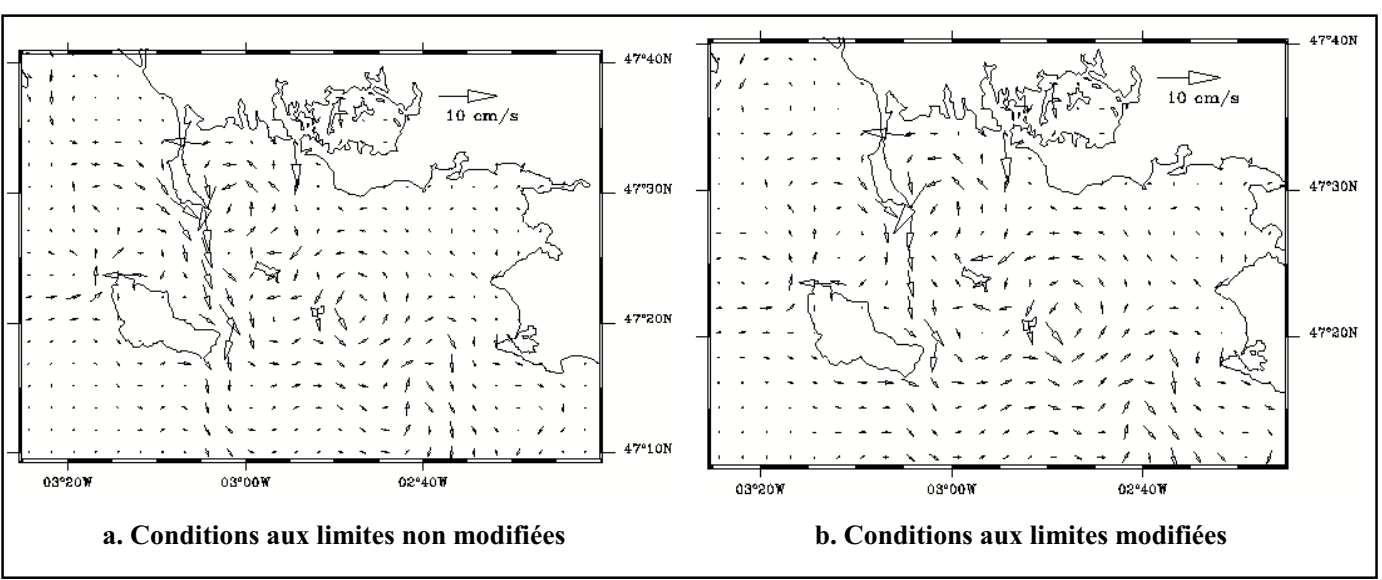

Figure 5 : Courants résiduels à la côte (modèle de « penbé ») 


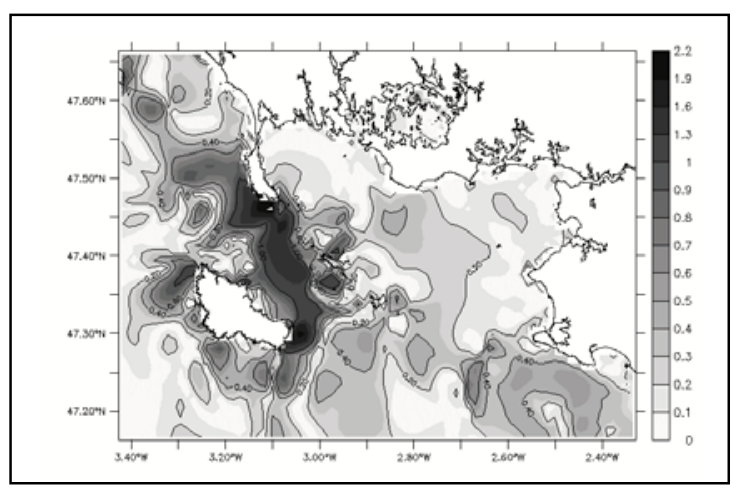

Figure $6:$ Ecart $(\mathrm{cm} / \mathrm{s})$ entre les courants résiduels à la côte (modèle de « penbé »)

On s'aperçoit alors que l'utilisation des données issues de CST France pour modifier les hauteurs d'eau calculées par le rang 0 perturbe la circulation résiduelle aux bords du rang de détail. Ce problème provient sans doute du fait que les hauteurs de marée issues de CST France résultent en partie d'interpolation de mesures et en partie d'un modèle numérique dont la bathymétrie n'est pas totalement compatible avec la

bathymétrie du modèle de rang 1 . Contrairement aux hauteurs calculées par le rang 0 , ces hauteurs d'eau ne correspondent donc plus forcément à la bathymétrie locale. Ceci peut générer des courants résiduels à certains endroits. En revanche, comme l'illustrent les figures 5 et 6 (modèle de Penbé), lorsqu'on se rapproche de la côte, le changement est assez peu sensible. On remarque seulement quelques zones très restreintes près des îles où l'écart est plus important. De plus, on a vérifié qu'il ne se propage pas non plus dans les modèles de rang supérieur (ici dans le rang 2).

\section{Conclusion}

Ce travail a permis de valider et de comparer le comportement des rangs de détails du modèle MARS 2D pour deux modes d'introduction de la hauteur de marée aux limites :

- La méthode traditionnelle de calcul des conditions aux limites utilisée dans MARS, c'est à dire l'utilisation de hauteurs calculées par emboîtement.

- L'utilisation de hauteurs de marée calculées par composition harmonique de constantes issues d'un modèle de marée plus fiable (CST France) pour corriger la composante astronomique des conditions aux limites calculées par emboîtement classique.

On a pu montrer l'intérêt de la seconde méthode pour améliorer considérablement la représentation de la marée instantanée dans les rangs de détail tout en conservant l'influence des conditions météorologiques. En effet, avec ces hauteurs d'eau modifiées, les modèles locaux testés évaluent beaucoup mieux la marée et permettent d'obtenir des surcotes similaires à celles qui sont obtenues avec des conditions aux limites traditionnelles. En revanche, contrairement à la méthode traditionnelle qui garantit l'utilisation de hauteurs d'eau adaptées à la bathymétrie, le forçage par composition harmonique génère des courants résiduels perturbés aux frontières. Cette perturbation se propage, plus ou moins loin selon les zones considérées, dans le rang de détail. Mais elle semble assez peu marquée à la côte et dans les rangs supérieurs. Elle ne remet donc pas totalement en cause la possibilité d'effectuer la substitution des hauteurs de marée aux limites. En revanche, elle exclut certainement son utilisation dans des modèles locaux de rangs supérieurs. 


\section{Bibliographie}

[1] Démerliac M.A., (1974). Calcul du niveau moyen journalier. Annales hydrographiques du S.H.O.M, $5^{\text {ème }}$ série, Vol. 2, fasc. 1 ;pp. 49-57.

[2] Lefèvre F., Lyard F.H., Le Provost C.et Schrama E.J.O. (2002). FES99: A Global Tide Finite Element Solution Assimilating Tide Gauge and Altimetric Information. Journal of Atmospheric and Oceanic Technology: Vol. 19, No. 9, pp. $1345-1356$.

[3] Le Roy R. et Simon B.(2003). Réalisation et validation d'un modèle de marée en Manche et dans le Golfe de Gascogne. Application à la réalisation d'un nouveau programme de réduction des sondages bathymétriques. Rapport technique, EPSHOM, Rapport $n^{\circ} 002 / 03$.

[4] Uppala S.M. et al. (2005). The ERA-40 re-analysis. Quart. J. R. Meteorol. Soc., Vol. 131, pp. 2961-3012. 